\title{
Alcohol health warnings can influence the speed of consumption
}

\author{
Lorenzo D. Stafford ${ }^{1} \cdot$ Joe Salmon ${ }^{1}$
}

Received: 13 June 2016 / Accepted: 3 October 2016 / Published online: 26 October 2016

(C) The Author(s) 2016. This article is published with open access at Springerlink.com

\begin{abstract}
Aim Recent research has shown that adopting strong (i.e. high fear) visual health-warning messages can increase the perceived health risks and intentions to reduce alcohol consumption. Separately, it is known that the speed at which alcohol is consumed has dramatic effects on the level of intoxication. In the present study we aimed to combine these two separate areas to understand whether the speed of alcohol consumption is influenced by the type of alcohol health warning contained on the beverage.

Subject and methods In the present study, female participants $(N=45)$ consumed an alcoholic beverage in a relaxed environment in one of three conditions: no health-warning label, a text-only health-warning label or a pictorial health-warning label with text.

Results We found that compared with the control condition, the beverage was consumed at a slower rate in the two healthwarning conditions, which surprisingly did not differ from each other. Despite these effects, product acceptability did not differ between the text-only and control conditions.

Conclusion These are the first set of results to demonstrate how the use of strong health warnings on alcoholic beverages can influence actual drinking rate and further suggest that the beneficial effects of slowed consumption are possible in the absence of any reduction in consumer acceptability.
\end{abstract}

Keywords Alcohol health warning · Alcohol consumption · Alcohol information

Lorenzo D. Stafford

lorenzo.Stafford@port.ac.uk

1 Department of Psychology, University of Portsmouth, King Henry Building, King Henry I Street, Portsmouth PO1 2DY, UK

\section{Background}

Alcohol is one of the most widely consumed drugs, integrated into the fabric of many cultures around the world; but this relationship comes at a price. In the UK alone, it is believed that 9 million adults drink to a level that poses health risks, with over 1.5 million individuals having some degree of alcohol dependence (Public Health 2014). This does not just impact the individual, it has bearings on society, and economics; alcohol-related harm is thought to have cost $£ 21$ billion in 2013-2014, with $£ 3.5$ billion of that cost related to alcohol issues treated via the National Health Service (NHS; Public Health 2014). Given these statistics, it is therefore imperative that strategies for curbing the excessive consumption of alcohol are considered and research engaged to examine their effectiveness. As part of this process, it is worthwhile learning any lessons from the huge change in public attitudes and behaviour to another widely consumed drug: Nicotine. In England, smoking has decreased from $39 \%$ of adults in 1980 to $20 \%$ in 2010 (HSCIC 2013). The factors responsible for this significant change are a combination that include health-warning labels on packaging, education on the damaging effects of smoking and smoke free legislation (Martin-Moreno et al. 2013). In terms of warning labels, research has shown that they are an important reminder on the danger of smoking and are strongly linked to intentions to stop smoking (Hammond 2006).

The effectiveness of health-warning labels in relation to tobacco have been examined with several factors being identified. Large warning labels are most effective, being large enough to be easily noticed and read (Hammond et al. 2007); Smokers report greater recall for warning labels on the front of packages, and that if 
the label had a direct and accurate message in conjunction with being simple, a greater impact is found (Hammond 2011); pictorial health-warning labels are more effective than text-only as pictures increase the accessibility to a greater target audience, whilst allowing smokers to visualise problematic scenarios (Hammond et al. 2004); also, colour pictures are more effective than black and white pictures.

In terms of alcohol, research has investigated public acceptability and consumer aspects of alcohol warning messages (e.g. Jarvis and Pettigrew 2013; MartinMoreno et al. 2013; Thomson et al. 2012), but very little work has examined whether such warning messages can actually change attitudes and virtually none looking at behavioural change. One Internet-based study found that when confronted with choices of which beverage to select with warning messages, young drinkers showed a reduction in choosing the alcoholic beverage (Jarvis and Pettigrew 2013). Work has also demonstrated that advertisements with fear arousing alcohol related visuals (e.g. ambulance at crash scene caused by excess alcohol) led to increases in perceived risks associated to alcohol use (Slater et al. 2002). Theoretically, those findings supported the Extended Parallel Processing Model (EPPM, De Hoog et al. 2007) which predicts that messages accompanied by threatening visuals induce more processing of the accompanying message. Most recently, we conducted an experiment (Wigg and Stafford 2016) where individuals were exposed to alcoholic beverages in three separate conditions (no health warning, text-only, pictorial), and found that participants exposed to the pictorial condition had higher intentions to reduce and quit alcohol consumption; which was related to individuals level of fear arousal.

The aforementioned study provided a useful first step in establishing experimental protocols in this domain; however, it was unclear from that work alone if such health-warning labels can influence other behavioural measures related to alcohol consumption. One such measure is the speed at which alcohol is consumed, which is important since the same quantity of alcohol drunk in a short versus longer period of time will have dramatically different effects and thereby risks associated to individual health and safety (Moskowitz and Burns 1976; BernoskySmith et al. 2012). Episodic drinking rate is driven by a number of factors including the sensory (taste) aspects of the drink that cue the presence of alcohol strength and the perceived behavioural (intoxicating) effects of alcohol (Higgs et al. 2008). It would also seem probable that speed of consumption is related to 'protective drinking behaviours' (Ray et al. 2009); the extent to which individuals engage in practices such as pacing the number of drinks consumed in a time period. Work has shown that individuals who score higher in protective drinking behaviours also have fewer negative consequences of alcohol consumption (Ray et al. 2009), which reinforces the point regarding the importance of the rate at which alcohol is consumed.

The current study therefore aimed to examine whether health-warning messages have the ability to influence this important aspect of alcohol behaviour. To test this question, individuals consumed the same alcoholic beverage in one of three separate conditions that varied in the type of health-warning on the bottle: no health-warning label; text-only; pictorial and text health-warning label. We tentatively predict that consumption rate will be fastest in the 'no health-warning label' category compared to both textonly and pictorial conditions, with the latter predicted to have the slowest rate.

\section{Methods}

\section{Participants}

Forty-five female university students, aged between 18 and 25 years $(M=18.93, S D=1.12)$ participated in this study. Participants were recruited using an online system and via social media with the study advertised as 'factors involved in alcohol related behaviour'. Participants were invited to take part only if they were aged between 18 and 25 years, were female and regular consumers of alcohol (see AUQ). All participants were in good health and to our knowledge free of any somatic diseases. The study protocol was given ethical approval from the department's ethics committee (British Psychology Society guidelines, similar to the Declaration of Helsinki).

\section{Design}

A between-subjects design was used, where participants were randomly allocated to one of three groups based on the health-warning label condition: control (no healthwarning label), text only health-warning label, or pictorial health-warning label with text (Table 1). The duration to consume the test beverage was the main dependent variable. 
Table 1 Mean (SEM) participant characteristics

\begin{tabular}{|c|c|c|c|c|c|c|c|}
\hline & \multicolumn{7}{|c|}{ Group } \\
\hline & \multicolumn{2}{|c|}{ Control } & \multicolumn{2}{|c|}{ Text only } & \multicolumn{2}{|c|}{ Pictorial } & \multirow[t]{2}{*}{ Group differences } \\
\hline & M & SE & M & SE & $\mathrm{M}$ & SE & \\
\hline Age & 18.9 & 0.3 & 18.8 & 0.3 & 19.1 & 0.2 & $F=0.36, \mathrm{NS}$ \\
\hline UK alcohol units (p/week) & 16.1 & 2.4 & 15.5 & 2.8 & 12.9 & 1.4 & $F=0.56, \mathrm{NS}$ \\
\hline MAST & 0.8 & 0.2 & 0.3 & 0.1 & 0.7 & 0.2 & $F=2.14, \mathrm{NS}$ \\
\hline PB-total & 8.7 & 0.4 & 9.2 & 0.5 & 2.1 & 0.5 & $F=0.35, \mathrm{NS}$ \\
\hline PB-pacing & 10.1 & 0.6 & 11.5 & 0.7 & 9.5 & 0.9 & $F=1.96, \mathrm{NS}$ \\
\hline PB-setting limits & 7.8 & 0.9 & 8.1 & 1.1 & 9.5 & 1.3 & $F=0.69, \mathrm{NS}$ \\
\hline PB-social & 10.8 & 0.3 & 10.3 & 0.4 & 10.7 & 0.2 & $F=0.78, \mathrm{NS}$ \\
\hline PB-diluting & 6.0 & 0.9 & 7.0 & 0.8 & 6.3 & 0.7 & $F=0.35, \mathrm{NS}$ \\
\hline No. of smokers & 1 & & 1 & & 0 & & \\
\hline
\end{tabular}

\section{Materials}

Alcohol usage questionnaire (AUQ)

Habitual alcohol consumption patterns were measured using a questionnaire (based upon Mehrebian and Russell 1978). Participants were accepted into the study only if their total weekly alcohol consumption was between 2 and 40 units of alcohol, consistent with previous research (Higgs et al. 2008; Stafford and Dodd 2013). Additionally, since the test beverage was vodka based, it was mandatory that participant's habitual alcohol consumption included vodka-based beverages to avoid compromising the validity of the study.

\section{Protective behaviours}

The extent to which individuals used protective behaviours whilst drinking alcohol was measured using the Protective Behaviours questionnaire (Ray et al. 2009). The questionnaire comprises 16 questions, e.g. 'I pace my drinks to 1 or fewer per hour'. Participants respond using a five point Likert scale ranging from Always true to Never true. The questionnaire yields scores in four different categories: pacing, setting limits, social, diluting.

\section{Michigan alcohol screening test (MAST)}

Participants were required to complete the MAST test before participating in the study. In order to avoid using any individuals with existing or potential alcohol related problems, individuals were only included in the study if they scored No apparent problem (0-2) on the 22 question test.

\section{Health-warning label}

The health-warning labels were based on Wigg and Stafford's (2016) design. The text-only health-warning label comprised of the statement 'Alcohol causes fatal liver cancer'. The same text was used for the pictorial healthwarning label with an image of a diseased liver. The health-warning labels were placed on the front of the alcoholic beverage as Hammond (2011) found that for smokers, greater recall was present when labels were on the front of packages.

\section{Product design questionnaire}

Before the study began, participants had to complete a pre-test product design questionnaire of the test beverage. This consisted of five questions on the acceptability of the bottle used (see Appendix). This was to ensure that participants actively observed the bottle and the healthwarning label, if present. Responses were recorded on a seven-point Likert scale, with 1 indicating the highest negativity and 7 referring to the most positivity towards the question.

\section{DVD programme}

As with previous similar research (Higgs et al. 2008; Stafford and Dodd 2013), in order to provide a more naturalistic environment and divert attention away from the real aim of the study, participants watched DVDs: Planet 
Earth, Jungles (Fothergill 2006, Planet Earth, Jungles, BBCDVD). The documentary was chosen as it is relatively neutral in tone and unlikely to induce different mood states, which may have affected the study.

\section{Blood alcohol level (BAL) readings}

Blood alcohol level readings were taken pre-test and poststudy via a breathalyser (Alcosens DA5000 digital). Participants could only partake in the study if their initial reading was a score of 0 . This reading was taken again post-study to determine if participants were safe to leave the administration room.

\section{Drinks and administration}

A pilot study was conducted to find the most suitable beverage to use in the study. Participants $(n=10)$ completed a questionnaire on the level of consumption and preference for a number of popular beverages. The drink with the highest ratings was 'WKD' (4\% ABV) and therefore used in the study. For the actual study, participants were given a $275-\mathrm{ml}$ bottle of freshly opened WKD served at fridge temperature.

\section{Procedure}

All testing took place between 11.00 a.m. and 5.00 p.m. in test rooms at the psychology department of the university. Upon arrival, participants provided informed consent, completed a breathalyser test using a digital personal breathalyser (all readings scored zero) and then completed the MAST, AUQ, and Prevention methods questionnaire. Next, the participants completed the pre-test product design questionnaire. Participants were then instructed to consume all of the drink while watching the DVD in their own time. The duration to consume the beverage was covertly timed by the experimenter using a mobile phone; with the timing commencing when participants took their first sip and stopped when they put the empty bottle back on the table. Participants then completed a post-test product design questionnaire to ensure participants were able to recall the health-warning information (if present). Their BAL was again measured. Each participant was given a partial debrief on the purpose of the study, and told that a full debrief would be sent out by email once all participants completed the study. This was felt necessary because a full debrief may have compromised the study for future participants. For safety reasons, participants were asked to wait in a waiting area for a period of time to minimise any adverse effects of the beverage, and informed not to operate heavy machinery or drive a vehicle for at least $3 \mathrm{~h}$. Course credit was administered for participation.

Analysis

The alcohol consumption rate was analysed using a univariate ANOVA, with the between-subjects factor of the group (no health-warning label/text only health-warning label/pictorial health-warning label with test). For the five product design questions, we completed reliability analyses and removed one item ('feel') with relatively low inter-item correlation, which resulted in a high Cronbachs alpha $(\alpha=0.76)$. The remaining four questions were summed together (termed 'acceptability') and analysed using a univariate ANOVA, with the betweensubjects factor of group (no health-warning label/text only health-warning label/pictorial health-warning label with test). Subsequent post-hoc tests were completed with Bonferroni adjustments.

\section{Results}

\section{Consumption duration}

Analysis revealed a significant effect of health-warning label, $F(2,42)=22.25, p<.001, \eta_{\mathrm{p}}{ }^{2}=.514$, where consumption was significantly faster in the "no health warning' compared to both the text and pictorial conditions $(p<.001)$, with the latter two not differing significantly $(p=.76$; Fig. 1). This suggests that the presence of a health-warning label is more important in influencing the rate of consumption than the type of message (text, pictorial).

\section{Acceptability of the alcohol beverage}

A significant effect of health-warning label was found, $F(2,42)=7.01, p=.002, \eta_{\mathrm{p}}{ }^{2}=.25$, with as expected, significantly lower ratings in the pictorial condition versus the 'no health warning' condition $(p=.002)$, but more surprisingly, there was no difference between the latter and the text condition $(p=.10)$. There were also no differences between the text and pictorial conditions $(p=.41)$. This suggests that although health-warning text can yield a decrease in the speed of consumption, there is no corresponding decline in the acceptability of the alcohol beverage (Fig. 2).

\section{Correlations}

In order to explore further the influences of the consumption rate, we computed correlations for the two 
Fig. 1 Mean duration of drinking as a function of health-warning label

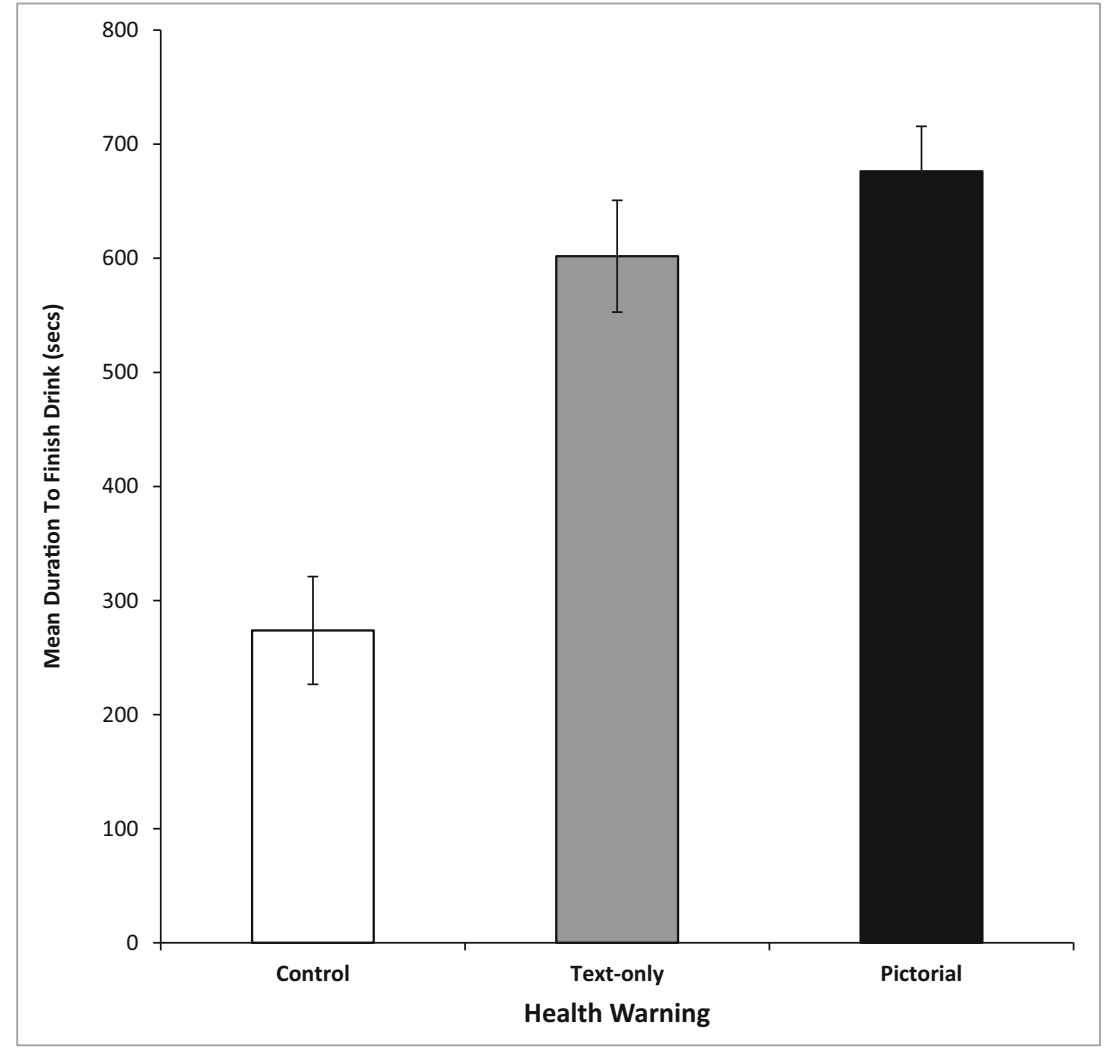

health-warning label conditions, between the duration to finish the drink and protective behaviours, product acceptability scores. None of these analyses were significant (Table 2).

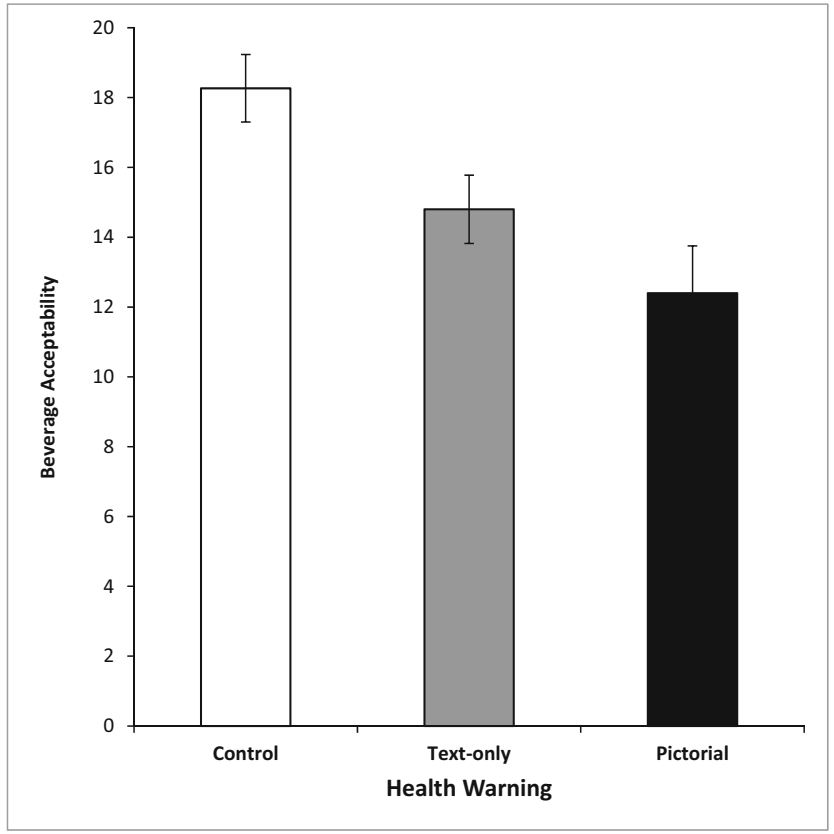

Fig. 2 Total acceptability ratings as a function of health-warning label

\section{Discussion}

The main finding of the study was that alcohol was consumed at a faster rate for those in the control condition compared to both the pictorial health-warning label and text only conditions. This pattern of findings is generally consistent with prediction and extends previous research (Wigg and Stafford 2016) to demonstrate that healthwarning labels can also influence episodic drinking behaviour. The mechanism responsible for slower consumption is theorised to be due to higher levels of fear arousal in the two health-warning conditions. It is well established that health-warning messages that elicit fear can be an effective means of informing the public and also instrumental in intentions to quit smoking (Kees et al. 2010).

Table 2 Correlations between consumption speed, protecting behaviours and product acceptability (text-only, pictorial conditions, $N=30$ )

\begin{tabular}{lr} 
Measure & Correlation \\
\hline Protective behaviours: pacing & 0.01 \\
Protective behaviours: setting limits & 0.01 \\
Protective behaviours: social & -0.28 \\
Protective behaviours: diluting & 0.18 \\
Acceptability & -0.09
\end{tabular}


For alcohol, it is also supported by work showing that individuals have less preference for alcohol products with health warnings (Jarvis and Pettigrew 2013). Hence, the lower preference for alcoholic beverages with health warnings may be manifested in the present study as a slower speed of consumption.

The observation that consumption speed did not differ between the pictorial and text-only conditions was surprising and contrary to prediction. In the previous study (Wigg and Stafford 2016), we found higher levels of fear in the pictorial versus text condition which made it seem probable to expect a slower consumption in the pictorial condition. This would also be consistent with the Extended Parallel Processing Model (EPPM, De Hoog et al. 2007) where health messages accompanied by threatening visuals have larger effects, as found in both alcohol (Slater et al. 2002) and tobacco research (Hammond et al. 2004; Schneider et al. 2012). However, it needs to be emphasised that those studies used different outcome measures from the study here, including perception of risk (alcohol) and intentions to reduce or quit (smoking). It could be therefore that the differences between pictorial and text-only health warnings are less important when measuring speed of consumption. Alternatively, it could be that the rate at which alcohol is consumed is not sufficiently sensitive to capture these differences and that adopting a different measure such as volume of consumption might be more appropriate. Connected to this, one study has found that the amount of alcohol consumed is sensitive when assessing the effectiveness of cognitive restraint training (Jones et al. 2011).

Given the aforementioned, the finding that pictorial health-warning labels led to lower acceptability ratings was not surprising; this is also consistent with work demonstrating that a cigarette's brand appeal can be reduced in the presence of health-warning labels (Thrasher et al. 2007). More interestingly was the finding that despite a clear difference in consumption speed, there were no corresponding difference in acceptability between the text and control conditions, which suggests that many of the advantages of including a health warning are available in the absence of any strong reductions in product acceptability. These findings also extend a previous study where alcohol warnings related to cancer did not provoke overly negative reactions (Pettigrew et al. 2014).

The absence of a relation between protective drinking behaviour and consumption rate is somewhat surprising. Since one of the components of that measure specifically relates to the habitual practice of pacing drinks, it seemed reasonable to assume that this would relate to actual speed of consumption. Previous research using the protective behaviour instrument found it had good accuracy in predicting future alcohol consumption over a given time period (Ray et al. 2009), but we are not aware of any work that examined its possible link with episodic drinking rate. It therefore seems likely that protective behaviours relate more to longer periods of self-reported alcohol consumption.

Considering the wider implications of the present study, since the speed of alcohol consumption influences the level of intoxication and associated health risks (Moskowitz and Burns 1976; Bernosky-Smith et al. 2012), the findings here have particular resonance; using such health-warning labels can help inform the public and could lead to safer drinking practices. These findings also have implications for wider alcohol work that has examined the drivers of consumption rate in terms of environment (e.g. music, Stafford and Dodd 2013) and sensory/ behavioural changes (Higgs et al. 2008). The present study extends those areas of research to show that consumption rate is also sensitive to the information presented on the alcohol product.

In terms of study limitations, due to the fact that this was a female only study, we cannot be sure if the impact of health warnings would be the same for male participants. The rationale to use females was based on the likely confound of consumption rate differences between genders and also the difficulty in finding an alcohol beverage consumed widely by both males and females; the use of one gender is also consistent with previous consumption rate research, (Higgs et al. 2008; Stafford and Dodd 2013). Interestingly, one might speculate that the effects observed in the present study would be weaker for males, based on the fact that work has shown that in response to alcohol health-warning advertisements, females responded more positively than males (Slater et al. 2002).

In summary, we found that individuals consumed alcohol more slowly when it was presented with pictorial or text-only health warnings. This is the first study to demonstrate this outcome measure in the context of health-warning messages similar to those used in the tobacco industry. The findings here suggest that using health warnings on alcoholic beverages could be an effective strategy in changing attitudes and behaviour to one of our favourite but harmful drugs.

\section{Compliance with ethical standards}

Ethical approval All procedures performed in studies involving human participants were in accordance with the ethical standards of the institutional and/or national research committee and with the 1964 Helsinki declaration and its later amendments or comparable ethical standards. 
Informed consent Informed consent was obtained from all individual participants included in the study.

Funding This study was funded by the University of Portsmouth

Conflict of interest The authors declare that they have no conflict of interest.

\section{Appendix: Product design questionnaire}

1. How pleasing do you find the design of the alcohol beverage?<smiles>C1CCC1</smiles>

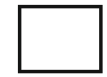

Not at all pleasing

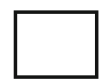

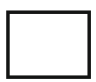

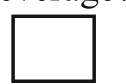

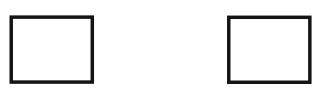

Extremely pleasing

2. How professional do you find the design of the alcohol beverage?<smiles>C1CCC1</smiles><smiles>C1CCC1</smiles>

Not at all professional
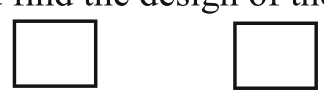
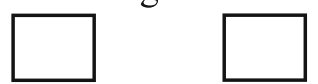

Extremely professional

3. How eye catching are the colours used on the design of the alcohol beverage?
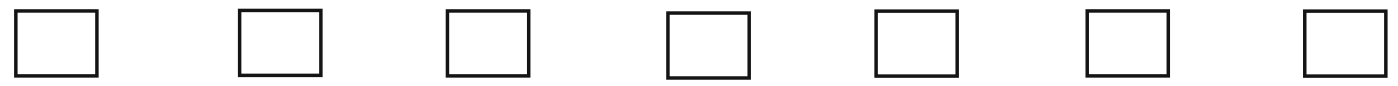

Not at all eye catching

Extremely eye catching

4. How important to you is the feel of an alcohol beverage in a glass or bottle/can?
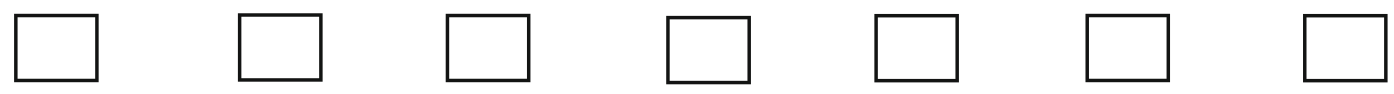

Not at all important

Extremely important

5. What is the likelihood of you purchasing this alcohol beverage based on its design?<smiles>C1CCC1</smiles><smiles>C1CCC1</smiles><smiles>C1CCC1</smiles>
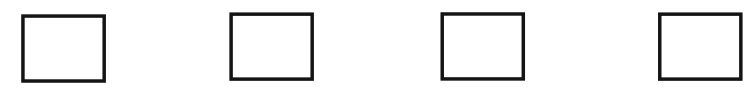

Not at all likely

Extremely likely 
Open Access This article is distributed under the terms of the Creative Commons Attribution 4.0 International License (http:// creativecommons.org/licenses/by/4.0/), which permits unrestricted use, distribution, and reproduction in any medium, provided you give appropriate credit to the original author(s) and the source, provide a link to the Creative Commons license, and indicate if changes were made.

\section{References}

Bernosky-Smith KA, Aston ER, Liguori A (2012) Rapid drinking is associated with increases in driving-related risk-taking. Hum Psychopharmacol Clin 27(6):622-625

De Hoog N, Stroebe W, de Wit JB (2007) The impact of vulnerability to and severity of a health risk on processing and acceptance of fear-arousing communications: a meta-analysis. Rev Gen Psychol 11(3):258

Fothergill A (2006) Planet earth: jungles [DVD]. BBC Natural History Unit, Bristol, UK

Hammond D (2006) Effectiveness of cigarette warning labels in informing smokers about the risks of smoking: findings from the international tobacco control (ITC) four country survey. Tob Control 15(3):19-25. doi:10.1136/tc.2005.012294

Hammond D (2011) Health warning messages on tobacco products: a review. Tob Control 20(5):327-337. doi:10.1136/tc.2010.037630

Hammond D, Fong GT, McDonald PW, Brown KS, Cameron R (2004) Graphic Canadian cigarette warning labels and adverse outcomes: evidence from Canadian smokers. Am J Public Health 94(8):14421445. doi:10.2105/ajph.94.8.1442

Hammond D, Fong GT, Borland R, Cummings KM, McNeill A, Driezen P (2007) Text and graphic warnings on cigarette packages. Am J Prev Med 32(3):202-209. doi:10.1016/j.amepre.2006.11.011

Higgs S, Stafford LD, Attwood AS, Walker SC, Terry P (2008) Cues that signal the alcohol content of a beverage and their effectiveness at altering drinking rates in young social drinkers. Alcohol Alcohol 43(6):630-635. doi:10.1093/alcalc/agn053

Jarvis W, Pettigrew S (2013) The relative influence of alcohol warning statement type on young drinkers' stated choices. Food Qual Prefer 28(1):244-252

Jones A, Cole J, Goudie A, Field M (2011) Priming a restrained mental set reduces alcohol-seeking independently of mood. Psychopharmacology (Berl) 218(3):557-565

Kees J, Burton S, Andrews C, Kozup J (2010) Understanding how graphic pictorial warnings work on cigarette packaging. J Public Policy Mark 29:265-276
Martin-Moreno JM, Harris ME, Breda J, Møller L, Alfonso-Sanchez JL, Gorgojo L (2013) Enhanced labelling on alcoholic drinks: reviewing the evidence to guide alcohol policy. Eur J Public Health 23(6): 1082-1087

Mehrebian A, Russell JA (1978) A questionnaire measure of habitual alcohol use. Psychol Rep 43(3):803-806. doi:10.2466 /pr0.1978.43.3.803

Moskowitz H, Burns M (1976) Effects of rate of drinking on human performance. J Stud Alcohol 37(5):598-605

Pettigrew S, Jongenelis M, Chikritzhs T, Slevin T, Pratt IS, Glance D, Liang W (2014) Developing cancer warning statements for alcoholic beverages. BMC Public Health 14(1):786. doi:10.1186/14712458-14-786

Public Health England (2014) Alcohol treatment in England 2013-14. http://www.nta.nhs.uk/uploads/adult-alcohol-statistics-2013-14commentary.pdf. Accessed 20 October 2015

Ray AE, Turrisi R, Abar B, Peters KE (2009) Social-cognitive correlates of protective drinking behaviors and alcohol-related consequences in college students. Addict Behav 34(11):911-917. doi:10.1016/j. addbeh.2009.05.016

Schneider S, Gadinger M, Fischer A (2012) Does the effect go up in smoke? A randomized controlled trial of pictorial warnings on cigarette packaging. Patient Educ Couns 86(1):77-83

Slater M, Karan D, Rouner D, Walters D (2002) Effects of threatening visuals and announcer differences on responses to televised alcohol warnings. J Appl Commun Res 30(1):27-49

Health and Social Care Information Centre (2013) Statistics on smoking: England, 2013. http://digital.nhs.uk/catalogue/PUB11454/smokeng-2013-rep.pdf. Accessed 20 October 2015

Stafford LD, Dodd H (2013) Music increases alcohol consumption rate in young females. Exp Clin Psychopharmacol 21(5):408-415. doi:10.1037/a0034020

Thomson LM, Vandenberg B, Fitzgerald JL (2012) An exploratory study of drinkers views of health information and warning labels on alcohol containers. Drug and alcohol review 31(2):240-247

Thrasher JF, Rousu MC, Anaya-Ocampo R, Reynales-Shigematsu LM, Arillo-Santillán E, Hernández-Ávila M (2007) Estimating the impact of different cigarette package warning label policies: the auction method. Addict Behav 32(12):2916-2925. doi:10.1016/j. addbeh.2007.05.018

Wigg S, Stafford LD (2016) Health warnings on alcoholic beverages: perceptions of the health risks and intentions towards alcohol consumption. PLoS One 11(4):e0153027 